\title{
Cognitive pitfalls in dealing with sustainability
}

Philipp Sonnleitner, Luxembourg Centre for Educational Testing, University of Luxembourg

\section{Main summary points:}

- Environmental conditions in which human ancestors evolved, strongly shaped human thinking

- Two complementary cognitive systems can be distinguished whose interplay causes systematic errors, especially in situations including uncertainty or complexity

- These cognitive pitfalls have tremendous consequences in the context of sustainability

- Leveraging cognitive biases and knowledge about them by improving education and training is vital to address ecological problems

\section{The challenge}

The human mind developed to make sense of the world, to structure it, and hence, control it as far as possible to maximize a human’s chance of survival (Geary, 2005). The way it works was optimized for the environment in which the genus Homo was evolutionary shaped, a time period roughly ranging from 2.6 million years ago until 12.000 years ago, also called the Paleolithic (Wood, 2005). This so called environment of evolutionary adaptedness, however, is foundationally different from the world of today. Instead of being overwhelmed by easily available food choices in the local supermarket, early humans were struggling to secure food at all. Instead of dealing with long term risks of instant pleasures like a decent meal or smoking a cigarette, they dealt with immediate threats that could only be solved by quick, physical decisions like running for their life. Instead of living in a complex, interconnected world, in which every action has (often unintended and unforeseeable) side 
and long-term effects on a local as well as on a global level, small social clans were formed in which actions had immediate consequences and problems were just locally relevant and comparatively straightforward. In short, large parts of the brain were optimized for a world that was completely different from today’s.

This chapter discusses how this cognitive heritage is influencing human decision making, especially in the context of sustainability. First, the theoretical framework for this endeavor is set by introducing dual-process models, the current approach in cognitive psychology to describe human thinking. On base of these models, typical errors in human decision making, such as framing effects, temporal discounting, or problems in dealing with complex systems are explored, and linked to the environmental context. This chapter closes by reinterpreting these systematic errors as part of the solution, and discussing how existing knowledge about the way human thinking works could be leveraged for solving the numerous challenges today's society faces.

\section{Human thinking through the lens of cognitive psychology}

Phenomena of human decision making are best studied and described by using a (cognitive) model that formalizes and at the same time structures our conception of how the brain works. To this end, this chapter adopts the theoretical framework of cognitive psychology which offers a wealth of studies enlightening the reasons for cognitive fallacies in everyday decision making and complex problem solving. Mainly drawing on an experimental approach, cognitive psychology tries to understand and adequately describe how people perceive information, process it and hence, act upon it (e.g. Solso, 2001). Although resulting theoretical models can best be understood as approximations of human thinking processes, 
they enable a glimpse into what is going on in the mind and allow for structuring and predicting complex cognitive performances, such as problem solving, reasoning, or reading.

Current approaches to describe human thinking are called dual-process theories and mainly distinguish between two cognitive systems (e.g. Evans \& Over, 1996; Sloman, 1996; Stanovich \& West, 2000). The first one encompasses so-called “cognitive modules” that evolved relatively early and enabled individuals to make faster and beneficial choices given the ecological context they lived in (Geary, 2005). Preferring high caloric, sweet or fat food that delivers more energy compared to other types of nutrition, would be an example for such a "cognitive module” (e.g. Beidler, 1982). Since functioning of these modules is largely unconscious, and automatic, they are often summarized using the umbrella term Autonomous Set of Systems, Implicit System or System 1 which is optimized to respond to immediate cues or threats (Evans, 2003; Kahneman, 2011; Stanovich, 2009). The second system, usually called Analytic System, Explicit System or System 2, in contrast, evolved relatively late; first indications of it are found in cave drawings from the Upper Paleolithic about 60.000 to 30.000 years ago, demonstrating that some form of abstract thinking was possible, allowing for symbolizing a bear by an abstract image painted on a cave wall. System 2 encompasses conscious thinking processes and allows humans to reason, and to plan by extending the time perspective and mentally simulating cause-and-effect chains. These complex performances, however, are limited by working memory and are therefore slower than cognitive modules of System 1. System 2 is therefore more prone to errors and causes a higher metabolism of the brain which often leads to actions based on "intuitive” suggestions of System 1 instead of carefully planned outcomes (Evans, 2003; Geary, 2005; Kahneman, 2011; Stanovich, 2009). Depending on the situation, the two cognitive systems are differentially suited (Geary, 2005). Choosing the most appropriate credit rate is something that should not be decided by your gut feelings which are product of System 1. Social situations, in turn, might quickly 
become awkward when your vis-à-vis’ body posture, facial expression, and voice intonation is overthought too much by System 2. Problems in decision making start when situations have cues that trigger the automatic System 1 but should better be approached by the conscious System 2, leading to irrational and suboptimal solutions.

\subsection{Merits and limitations of the chosen theoretical approach}

Especially when talking about psychological phenomena, it has to be kept in mind that no model is identical with what it aims to describe, but is more like a simplified image of it. The same applies for cognitive models of human thinking, which often use a highly mechanistic account and strongly shape the way how we think and do research about certain topics. Further, a short glance at the history of cognitive psychology shows that such models mostly are temporary aids to describe certain phenomena of thinking and are modified or replaced after some decades (e.g. Solso, 2001). Whether dual-process theories stand the test of time remains to be seen but they face several criticisms, including convincing alternative approaches, unifying all cognitive processes within one coherent system (cf. Evans \& Stanovich, 2013 or Osman, 2004, 2014).

Interestingly, one reason for the simplicity of existing models to describe the human mind can be seen in the mind itself. As will be discussed later in the chapter, people have considerable problems in dealing with complexity, and interpreting or using cognitive models to explain human behavior is no exception. However, one reason for the development of System 2 was the need to explain what is going on in the world by building abstract models that are used to explain and hence, control the environment (Geary, 2005). Thus, applying somewhat inaccurate but useful working metaphors to guide actions and make predictions is typically human. 
Note that research on cognitive biases, fallacies, or pitfalls has already had tremendous impact by disenchanting the myth of humans as economically rational actors (Kahneman, 2003, 2011). If society strives for a sustainable development, however, it has to be even more aware of the implications of these cognitive biases on everyday decision making. Not only do they explain why people behave in an irrational and unsustainable way in the first place, but knowledge of them also offers possible solutions for avoiding or counteracting | them in the future. In the following, it will be demonstrated how this “cognitive architecture” of the brain leads to a variety of fallacies when people are confronted with decisions.

\section{Cognitive fallacies in decision making}

\subsection{Prospect theory and framing effects}

Depending on whether the focus is set on gains or losses, people opt for different choices, an (irrational) behavior first explained by Kahneman and Tversky’s (1979) prospect theory. The credit card industry, for example, made sure that any surcharge for paying with card was relabeled as discount for paying in cash. Losses are usually perceived to weigh heavier than gains, therefore paying a surcharge for using a credit card would be much less attractive than simply to forgo the benefit of paying in cash (see Thaler, 1980). Thus, the way a problem is framed, that is how a certain issue is introduced and presented, heavily influences how people see, understand, and decide on that problem (Kahneman, 2003). Several studies consistently demonstrated that people seek risk when the focus of a problem is set on sure losses, and avoid risk when the focus is set on gains (see Box 1 for a typical example). Crucially, this pattern not only affects lay people, but also professionals in their domain of expertise. In the context of deciding between cancer therapies, McNeil, Pauker, 
Sox, and Tversky (1982) showed that even trained physicians’ choice heavily depended on the framing of the problem, avoiding risks when presented with sure gains and preferring risky options when confronted with unavoidable losses.

This discrepancy arises from two characteristics of System 1 (e.g. Stanovich, 2004). First, System 1 is biased to accept given information and the context or "frame" as is. Reformulating the initial propositions would be mentally effortful and System 2 would be needed. Second, the described utility function of preferring secure gains over more promising but risky options must have been advantageous in the environment of evolutionary adaptedness, true to the motto "a bird in the hand is worth two in the bush". Such behavior absolutely makes sense in an insecure environment in which resources are scarce. Since System 1 acts automatically and faster than System 2, when reading such scenarios as in Box 1, our gut feelings thus immediately give us a preference for a certain option. Note that when participants were told to directly compare both scenarios, in other words, System 2 was activated, all choice preferences between the scenarios vanished. Consequently, it's not that people weren’t able to figure out that the scenarios were in fact identical, they just gave the answer that first came to their mind. 


\section{Box 1 - Engaged Learning Activity: Framing Effects}

Read through Scenario 1 and decide which option you would take. Note your decision.

\section{Scenario 1:}

Imagine an African village with 600 inhabitants. You would like to ensure power supply for this village but given the specific circumstances (financial situation, local climate), there are only two alternatives:

A) If a diesel generator is purchased, the supply for 200 inhabitants is guaranteed.

B) If a hydroelectric power production is installed, there is a one-third probability that 600 inhabitants can be provided with energy and a two-third probability that due to a drought year, nobody has power.

Now read through Scenario 2 and again, note down your decision.

\section{Scenario 2:}

Imagine an African village with 600 inhabitants. You would like to ensure power supply for this village but given the specific circumstances (financial situation, local climate), there are only two alternatives:

$A^{`}$ ) If a diesel generator is purchased, 400 inhabitants will be without power supply.

$\mathrm{B}^{`}$ ) If a hydroelectric power production is installed, there is a one-third probability that everybody will have power supply and a two-third probability that due to a drought year, 600 inhabitants will be without power supply. 
This example is a typical problem used in research studies on framing effects and identical concerning the numbers to the example used in Kahneman (2003). Essentially, both scenarios are exactly the same, only in Scenario 1 focus is set on sure gains which render the uncertain, risky decision B less attractive whereas in Scenario 2, focus is set on sure losses, making the riskier decision B' more attractive to choose. In fact, this prediction by prospect theory has been confirmed in a huge amount of studies, reporting a majority of people choosing A in Scenario 1 and B` in Scenario 2.

\subsection{Framing in the environmental context}

It has been frequently shown that framing effects also play a central role within the context of sustainability. For example, Amelung and Funke (2015) investigated opinion formation concerning climate engineering techniques, such as cloud whitening or injecting aerosols in the stratosphere to reduce solar radiation reaching earth's surface, as an alternative to mitigating $\mathrm{CO}_{2}$ emissions when fighting global warming. They could show that participants focusing on the risks of current political efforts to mitigate $\mathrm{CO}_{2}$ emissions and doubting their success, were more likely to adopt climate engineering strategies than participants reporting more faith in more conservative approaches to the problem. Results clearly show that the framing of climate engineering techniques as a plan B or back-up strategy that is usually employed in related research proposals and media coverage, strongly shapes public opinion on the topic and henceforth steers the debate in a more benevolent direction towards these techniques (Amelung \& Funke, 2015; Bellamy, et al., 2012). Thus, risk framings, directly targeting certain features of System 1, can create powerful momentum to steer public and hence political opinion. A prominent example in this context can also be seen in the German nuclear phase-out in June 2011 that was a direct response to the 
Fukushima Daiichi nuclear disaster that happened 3 months earlier in Japan. Initially, the then ruling government led by Chancellor Angela Merkel had decided to significantly extend the operating time for German nuclear power plants only to decide on immediate shut downs and restrictions of operating time after the incident (Appunn, 2015).

Substantial framing effects were also found concerning the environmental discourse in the United States that is known to be highly polarized between liberals and conservatives (Feinberg \& Willer, 2013). Liberals’ stronger pro-environmental attitudes (e.g. McCright \& Dunlap, 2011) could largely be explained by the current framing of the environmental debate in terms of harm and care principles - moral concepts that were found to be more strongly embraced by liberals than by conservatives. Strikingly, if environmental messages were reformulated corresponding to moral values of purity and sanctity, differences in proenvironmental attitude between liberals and conservatives completely vanished. One suggested reason for this shift was that the purity/ sanctity framing referred to moral principles typically advocated by conservatives, thus triggering System 1 of the conservative participants to detect familiarity and hence, to put greater trust in the presented message. These findings not only demonstrate the strong impact of certain framings of environmental information and messages on public opinion but also highlight that framing effects can be general but also differential. Since messages or choices cannot be "unframed", it is especially important to critically reflect the frame that is used.

\subsection{Temporal discounting and climate change}

Risk or uncertainty, are also crucial when thinking about the future. Given the challenging and unpredictable setting, in which the genus Homo mainly developed, it comes as no surprise that System 1 is optimized to prefer instant gains over long-term rewards, a pattern also known as temporal discounting. Within the climate change context, the problem 
is even more aggravated by the fact that potential but uncertain benefits of immediate costs (e.g. significant reduction of resource consumption) do not only lie in the far future, they only become perceivable within the next generation(s) at best. Consequently, Jacquet and colleagues (2013) could impressively show that a common climate protection goal (the financing of a related newspaper ad), was reached in $70 \%$ of the participating groups when there was an instant (monetary) reward, in 36\% of the groups if there was a 7 week delay of their endowment, and in none of the groups if the common benefit was invested in planting trees, a proxy for an intergenerational advantage. Especially in the last condition, selfish behavior of group participants dominated throughout the experiment. According to the authors, international climate change negotiations won’t succeed until powerful short-term incentives, such as punishment, reward or reputation will be introduced to the debate, thus somewhat canceling the effects of temporal discounting.

Compared to stable and secure conditions, predictions for the future become even more uncertain when the environment is harsh. How this factor additionally influences decision making, was shown in a recent experiment by Laran and Salermo (2013) in which participants were primed with either neutral cues or cues of environmental harshness while having to choose between high-caloric or low-caloric food alternatives. In the environmental harshness condition, triggering primordial reactions of System 1, participants significantly chose high-caloric food over low-caloric alternatives, indicating that they (subconsciously) prepared for tough times by securing high energy food. Crucially, this preference vanished when neutral cues were presented, strongly suggesting that people adopt different strategies depending on the perceived environmental conditions. This relation has also been demonstrated in other studies, showing that the harsher and the more uncertain people perceive their personal future, the more likely they are to adopt a so-called fast life-history strategy that is associated with higher consumption, gambling, or a lower age at giving the 
first birth (Nettle, 2010; Griskevicius et al., 2012). In the light of these findings, however, it seems highly doubtful that current warnings of climate change's consequences, a looming scarcity of resources, and a constant environmental alarmism will have their envisaged effects. Instead, such messages might even increase non-sustainable behavior in that they trigger cognitive systems that are sensitive to such threats and urge people to think about their own advantage first.

\subsection{Dealing with problems that are complex}

Most problems in the context of sustainability are not isolated but arise within systems and networks and were thus described as complex problems (see also Chapter 3). Such problems typically incorporate five characteristics that define them as complex (e.g. Funke, 2001, Dörner, 1989): They (a) consist of a large number of elements that (b) are constantly influencing each other. The underlying relations (c) include dynamics, for example side effects or variables that, depending on their state, autoregressively change on their own and are (d) not transparent to the problem solver. In addition, most scenarios (e) require the problem solver to achieve several, partly contradictory goals. How these characteristics can be found in the (complex) problem of applying climate engineering is demonstrated in Box 2 .

Basically, interacting with or trying to control complex systems can be seen as a constant stream of decisions. Since cognitive capacities are limited, the first decision already starts with choosing where to draw attention within the system. If a problem is identified, appropriate strategies have to be selected and applied in order to gather knowledge on this issue, for example how a certain variable (e.g. the application of climate engineering) influences others (e.g. local temperatures, public opinion on this topic). The impact of interventions has to be evaluated though, and the decision on the right indicators (e.g. solar radiation, frequency of extreme weather phenomena) and the right time frame (e.g. months 
vs. years) has to be taken. If finally, it was decided which information is transferred into knowledge, this understanding has to be purposefully applied and choices concerning the right targets have to be made. However, since full knowledge of the system's underlying connections is mostly impossible as is the definition of goals that are optimal for all involved stakeholders, complex problem solving can be modelled as a sequence of decisions that are made under uncertainty (e.g. Osman, 2014). This, however, also implies that all phenomena discussed above, such as framing effects or prospect theory come into play.

\section{Box 2. Climate engineering as a complex problem?}

In an excellent paper on the uncertainties of climate engineering, Amelung and Funke (2013) illustrate how the decision on injecting aerosols in the stratosphere to reduce solar radiation and thus counter extreme weather events, can be described using criteria of the complex problem solving research paradigm:

(a) Complexity: A large number of variables have to be considered for informing this decision. Besides obvious physical and biological parameters, such as the expected change in local temperatures and weather conditions and its effects on the prevailing fauna and flora, also psychological, social, economic, legal, and hence, political aspects have to be considered. The application of climate engineering techniques might be refused by the public, but needs sufficient support of political decision-makers who have to negotiate this supranational issue also with neighboring states. A successful application might have varying effects on different economic branches (agricultural sector vs. tourism) and benefits may not outweigh deployment costs and related risks.

(b) Connectivity: The involved variables are part of a system and in a constant feedback-loop with each other. The prospect of a solution to climate change might lead to a less responsible consumer behavior neutralizing some of the positive effects. In addition, the 
technique itself, injecting aerosols in the stratosphere, would have several effects, indirect and probably, due to a lack of case-studies, also unknown ones on variables that are not yet considered.

(c) Dynamics: Even if the technique would successfully reduce solar radiation, the impact on temperature and local weather phenomena might not be linear in nature and thus, be delayed. This, however, may lead to a disappointed public putting increased pressure on policy makers who could then feel forced to intensify their efforts with even more drastic interventions.

(d) Intransparency: Although some climate parameters can approximately be reproduced in computer simulations, understanding the whole (itself complex) climate system is still beyond reach. Together with unpredictable social impacts, these unknown processes add to the intransparency of the decision's consequences.

(e) Polytely. The manifold and various effects of climate engineering would benefit all involved stakeholders differently. Consequently, multiple and partly contradicting goals have to be considered and weighted for this decision. Given the large number of involved factions with contradicting regional interests might render an optimal solution even impossible.

\subsection{Typical errors when dealing with complex systems}

Human performance in solving complex problems has been addressed in various disciplines, ranging from economics to cognitive psychology, using a broad variety of computer-based simulations that mirror dynamic and non-linear real-word scenarios (for a thorough overview see Osman, 2010). In general, by interacting with such scenarios over a certain amount of time, people could learn to control these systems reasonably well, with 
knowledge on the underlying connections being the key aspect (e.g. Beckmann \& Goode, 2010; Sonnleitner, Brunner, Keller, \& Martin, 2013; Osman, 2014). But research also revealed a significant amount of typical errors that are made.

If you know nothing about a system, the first step is to explore its underlying mechanics in order to build a mental model of how the variables are linked to each other. But where to turn first and how to find out? Most studies have revealed, that people don't apply a systematic way to gather knowledge, probably because no hypotheses are formulated and hence tested (Dörner, 1989). Partly, this could be explained with the lack of clear goal setting during this initial exploration phase. With the diffuse and uncertain aim of finding out how the scenario works, the mind looks for familiar anchors and System 1 "intuitively" suggests topics or problems that fit this criteria. Beckmann and Goode (2014) found out, however, that such "false familiarity" - vague but in no way perfect knowledge that is triggered by the semantic meaning of a certain system variable - has detrimental effects on knowledge acquisition. Existing assumptions are taken as correct and are not systematically tested, thus leading to a faulty or incomplete mental model of the scenario. The missing of a deliberately planned exploration strategy and the subsequent reliance on familiarity also leads to so-called "thematic vagabonding" and the focus on single goals instead of a necessary holistic problem-solving approach (Brehmer, 2005). This fragmentary system exploration is aggravated by the tendency to overestimate the status quo of a system - in other words, the information that is available right now - and overlook feedback delays or future side effects and tipping points of their interventions. Again, in a relatively uncertain environment, System 1's predisposition of a myopic time perspective may be advantageous, but not in the long run.

A recent study by Sonnleitner, König, and Sikharulidze showed that students within a course on sustainable development and social innovation had problems to interpret information created while exploring a problem correctly. Effects of an invisible system 
variable were either completely attributed to other visually represented system elements or accounted for twice by simply not differentiating them from other visible effects. Reasons for these errors were seen in a complex interaction of overemphasizing visually represented information (a feature of System 1) and the high cognitive workload to disentangle two sources of an effect (limitations of System 2). These findings also point to the high impact of reasoning ability on complex problem solving performance. Studies showed that even full information about the underlying connections does not guarantee perfect achievement of envisaged targets; it depends on what you make out of this knowledge (e.g. Goode \& Beckmann, 2010; Sonnleitner, Brunner, Keller, Martin, 2013). Other mental pitfalls that were found in this phase of knowledge generation are mistaking correlations with causation, the illusion of being able to control variables that are beyond the influence of the problem solver, and the preference to look for evidence that supports the preconceived model instead of disproving it, which would be mentally more demanding (Brehmer, 2005; Dörner, 1989). Taken together, people were found to apply suboptimal strategies to generate information on unknown systems and in addition, build wrong mental models by drawing wrong conclusions about this information either because of mental limitations to understand the generated information or because of mental shortcuts provided by System 1. Thus, the basis for systemic interventions in most cases is already flawed.

Since problem solvers are in a constant feedback loop with the systems they interact with, they can learn from their mistakes, adapt their faulty mental models, and reasonably reach their envisaged targets (Brehmer, 2005, Osman, 2014). Frequently, however, this feedback loop ends in a downward spiral, especially when due to wrong knowledge, interventions have unexpected outcomes, and hence increase uncertainty and emotional pressure to solve the situation. Typical reactions to reduce these negative emotions range from rash, pointless actions to extensive and paralyzing information seeking to fill in the 
existing knowledge gaps (Dörner, 1989). Even if meaningful interventions are made, their impact could be delayed due to an exponential change rate or initially neutralizing sideeffects. Most people, however, expect and act upon linear changes and therefore increase their activities in the absence of estimated effects. The focus on present states and the neglect of processes underlying a certain system, finally, leads to an oscillation of actions causing a constant over- and undershooting of targeted values.

\section{Leveraging insights from cognitive psychology}

\subsection{Informing and "nudging" the public}

In the light of the reviewed examples, it seems evident that cognitive biases play a crucial role in the context of sustainability. But what can be done to lessen their impact or prevent them in the first place? One important step is raising awareness of cognitive biases in the broad public (for an excellent example see Marshall, 2014) and disciplines other than (cognitive) psychology. With the introduction of behavioral economics to many curricula focusing on finance and economy for example, this slowly gets momentum. Since environmental issues can only be tackled by a multidisciplinary effort, knowledge on human decision making should also be part of other disciplines that train students for acting within complex systems (e.g. engineering, politics, or spatial planning).

Another route is the explicit consideration of cognitive biases in policy making and the design of so-called decision architectures, as already done in some contexts (Thaler \& Sunstein, 2008). founIn fact, for several years governments have been exactly to do this, by applying a soft paternalism that “nudges” people in a certain direction when confronted with decisions (for a detailed discussion of this account and examples see Thaler and Sunstein, 2008). However, despite being promising, this approach has been empirically challenged and 
is far away from being the key to all sustainability problems (Osman, 2014, 2015). It has to be complemented by a stronger inclusion of the wider public through a social dialogue, otherwise such "nudging” will provoke reactance in people, the unwillingness to comply with actually beneficial measures, just because behavioral freedom is seemingly threatened through their introduction (e.g. Miller, Lane, Deatrick, Young, \& Potts, 2007). Instead of patronizing the public, informing it on an equal footing about existing mental pitfalls, showing them and conjointly developing ways to face uncertainty by avoiding such fallacies and having faith that people take on this responsibility, may not only be more mature but also more likely to succeed (Osman, 2014). An idea that Ravetz (2006) conceived as post-normal science.

\subsection{Adapting educational curricula}

One consequence of studying human decision making in complex environments, was the request for adapting educational curricula by explicit training of systems thinking using computer-based scenarios (Dörner, 1989; Vester, 2012). People should not only be informed about cognitive pitfalls in dealing with complex problems but should make their own experiences in order to learn from them. The question arises, however, how the training simulations should be designed in order to maximize the learning outcome. Should they reflect real, specific scenarios or be formulated in a more abstract, general way? Funke (2006) highlighted the discrepancy in this context that the more detailed scenarios are, the more situation-specific and therefore useless for other scenarios the acquired knowledge will be. On the other hand, if very general and abstract scenarios are used for training, domaingeneral principles might be demonstrated but their usefulness for domain-specific situations might be questionable. In addition, Beckmann and Goode (2014) pointed out that scenarios which use meaningful labels for the included variables, thus being “semantically embedded”, 
might hamper learning about the underlying connections and dynamics since learners might have false prefixed associations with the scenario’s variables that are not tested systematically (see above). They consequently suggest that novel or abstract contexts might therefore be advantageous for learning general skills like systematically exploring relations between variables. If semantically embedded scenarios have to be used in order to teach specific knowledge, essential steps of the training would be the explication and hence, the consequent and systematic test of learners’ assumptions about the system. Beckmann and Goode also highlight that if concrete goals are given in the phase of exploring a system, people aim to reach them simultaneously by manipulating several input variables at the same time. Needless to say that such a mix of interventions makes it impossible to draw clear cut conclusions about cause-and-effect chains and thus, significantly reduces the gathered knowledge. The interventions might have been successful but the learner does not know why, hence does not develop a secure foundation of understanding the system and finally, develops (unjustified) overconfidence in his problem solving skills. From this point of view, existing computer-based simulations aiming to teach systems thinking, such as Vester's Ecopolicy (2011) that asks the learner to solve several problems of a fictitious state (e.g. poor educational level and health conditions of the population, high environmental pollution, or a faltering economy) within a few steps, might not reach their goals.

Studies on the training of systems thinking and complex problem solving are still scarce and produced mixed results. Krétzschmar and Süß (2015) showed that university students were able to extract general principles of system exploration by just interacting with several, heterogeneous scenarios over a certain amount of time. Although they outperformed peers without training in gathering knowledge about a new scenario, they could not use this advantage in reaching specific target values. Micheli (2016) used another approach to teach complex problem solving, by discussing characteristics of complex problems and its 
implications for interacting with them within a one hour lecture and afterwards assessing students' problem solving skills. However, the intervention showed almost no effect. A successful training approach was reported by Akcaoglu, Gutierrez, Hodges, and Sonnleitner (2016). They found that students enrolled in a course on how to design and basically program computer games significantly improved their performance to interact with a complex problem solving scenario, thus pointing to the possibility that also indirect training methods might be efficient. An approach in the context of sustainability education was recently presented by Sonnleitner, König, and Sikharulidze (under review), who confronted learners with a gamified problem-solving scenario of reduced complexity. While working on the problem, students took notes about experienced emotions which were then discussed within a lecture focusing on cognitive pitfalls in dealing with complexity. Data revealed that typical problems and reactions observed in more complex and larger scenarios (as discussed above) could be replicated even in this simpler simulation, and hence used for increasing the awareness and sensitivity of learners for their own mistakes and limitations. Exercises to reflect, for instance, on framing effects, such as the one presented in Box 1 could additionally help to make these phenomena more tangible.

The need for sensitizing students to cognitive biases and for training them in systems thinking on a larger scale, even by integrating these aspects into educational curricula, is beyond doubt, given its importance (outlined in Chapter 3) and by the frequent, severe mistakes people make when learning and applying it. Recent efforts to include skills relevant for systems thinking in educational curricula (e.g. Micheli, 2016; OECD, 2014) and curricula on sustainable development (Sonnleitner, König, \& Sikharulidze, under review) paint a promising picture. But a clear and convincing concept of how to teach systems thinking and which tools to use for it, is still missing. For the moment, informing about and demonstrating typical cognitive biases through experience using simulations in this context, seems to be a 
solid foundation for further developments. Only if decision-makers face their own limitations in the light of uncertainty, can they appreciate the complex nature of interacting with systems and avoid some of the mistakes mentioned above.

\section{Conclusion}

The present chapter set out to highlight the influence of humans' cognitive heritage on today's intricate decisions, especially in the context of sustainable development. Although in the reviewed examples, this heritage seems more like a burden than a rich inheritance, history has shown that overcoming barriers by creatively solving problems using newly invented tools and techniques is at the core of the human species. In this sense, utilizing insights of (the tool) cognitive psychology and orchestrating it with other techniques reviewed throughout this book might be a promising path to solve the problems of sustainability in the best tradition of mankind. 


\section{Questions for comprehension \& reflection:}

- Why are spontaneous preferences for certain decisions sometimes suboptimal?

- What could be unintended consequences of campaigns against global warming emphasizing that society is running out of time to make a change?

- What characterizes complex problems and what problems do people have in dealing with them?

- Observe yourself the next time you go grocery shopping. How many of your purchase decisions do you take consciously, how many of them are based on spontaneous impulses?

- Imagine, your local supermarket reorganizes its shelves and at the place of your favorite chocolate bar, you find a low calorie alternative. A bit irritated, you find your preferred one at the very bottom of the shelf that is hard to reach. How would you feel about this "nudging” towards healthier alternatives?

- What could be done to better prepare executives to deal with complex problems and what are potential reasons for resistance?

\section{Core readings:}

- Brehmer, B. (2005). Micro-worlds and the Circular Relation between People and their Environment. Theoretical Issues in Ergonomics Science 6 (1), pp. 73-93.

- Kahneman, D. (2003). A Perspective on Judgment and Choice: Mapping Bounded Rationality. American Psychologist 58 (9), pp. 697-720.

- Marshall, G. (2014) Don't Even Think About It: Why Our Brains Are Wired to Ignore Climate Change. Bloomsbury USA.

- Vester, F. (2012). The Art of Interconnected Thinking: Ideas and Tools for tackling with Complexity. Munich: MCB-Verlag 


\section{References}

Akcaoglu, M., Gutierrez, A.P., Hodges, C.B. \& Sonnleitner, P. (2017). “Game Design as a Complex Problem Solving Process”, in Zheng, R. \& Gardner, M. K. (eds.) Handbook of Research on Serious Games for Educational Applications. Hershey, PA: IGI Global, pp. 217-233

Amelung, D. \& Funke, J. (2013). Dealing with the Uncertainties of Climate Engineering: Warnings from A Psychological Complex Problem Solving Perspective. Technology in Society 35 (1), pp. 32-40. doi: 10.1016/j.techsoc.2013.03.001

Amelung, D. \& Funke, J. (2015) Laypeople's Risky Decisions in the Climate Change Context: Climate Engineering as a Risk-Defusing Strategy? Human and Ecological Risk Assessment 21 (2), pp. 533-559. doi: 10.1080/10807039.2014.932203

Appunn, K. (2015). The History Behind Germany's Nuclear Phase-out. Clean Energy Wire Factsheet. [online] URL: https://www.cleanenergywire.org/factsheets/history-behindgermanys-nuclear-phase-out

Beckmann, J. F. \& Goode, N. (2014). The benefit of being naïve and knowing it: the unfavourable impact of perceived context familiarity on learning in complex problem solving tasks. Instructional Science 42(2): 271-290

Beidler, L.M. (1982). “Biological Basis of Food Selection”, in Barker, L.M (ed.) The Psychology of Human Food Selection, Chichester, UK: England Ellis Horwood Limited, pp.3-15

Bellamy, R., Chilvers, J., Vaughan, N. \& Lenton, T. (2012). A Review of Climate Geoengineering Appraisals. WIREs Climate Change 3(6), pp. 597-615. doi: 10.1002/wCc.197 
Cog pitfalls 170312 JR

Brehmer, B. (2005). Micro-worlds and the Circular Relation between People and their Environment. Theoretical Issues in Ergonomics Science 6 (1), pp. 73-93. doi: $10.1080 / 14639220512331311580$

Dörner, D. (1989). Die Logik des Misslingens: Strategisches Denken in Komplexen Situationen. [The Logic of Failing: Strategic Thinking in Complex Situations. Hamburg: Rowohlt

Evans, J. St. B. T. (2003). In Two Minds: Dual-process Accounts of Reasoning. Trends in Cogntive Science 7, pp. 454-459

Evans, J. St. B. T. \& Stanovich, K. E. (2013). Dual-Process Theories of Higher Cognition: Advancing the Debate. Perspectives on Psychological Science 8 (3), pp. 223-241.

Evans, J. St. B. T. \& Over, D.E. (1996). Rationality in the Selection Task: Epistemic Utility versus Uncertainty Reduction. Psychological Review 103(2), pp. 356-363. doi: 10.1037/0033-295X.103.2.356

Feinberg, M. \& Willer, R. (2013). The Moral Roots of Environmental Attitudes. Psychological Science 24 (1), pp. 56-62. doi: 10.1177/0956797612449177

Funke, J. (2001). Dynamic Systems as Tools for Analysing Human Judgement. Thinking and Reasoning 7 (1), pp. 69-89. doi : 10.1080/13546780042000046

Funke, J. (2006). “Komplexes Problemlösen [Complex Problem Solving]” in Funke, J. \& Birbaumer, N. (eds.) Denken und Problemlösen. Enzyklopädie der Psychologie. Göttingen: Hogrefe, pp. 375-446

Geary, D.C. (2005). The Origin of Mind: Evolution of Brain, Cognition, and General Intelligence. Washington, DC: American Psychological Association

Goode, N. \& Beckmann, J.F. (2010). You Need to Know: There is a Causal Relationship between Structural Knowledge and Control Performance in Complex Problem Solving Tasks. Intelligence 38 (3), pp. 345-352. doi: 10.1016/j.intell.2010.01.001 
Griskevicius, V., Ackerman, J.M., Cantú, S.M., Delton, A.W., Robertson, T.E., Simpson, J.A., Thompson, M.E. \& Tybur, J.M. (2013). When the Economy Falters, Do People Spend or Save? Responses to Resource Scarcity Depend on Childhood Environments. Psychological Science 24 (2), pp. 197-205. doi: 10.1177/0956797612451471

Jacquet, J., Hagel, K., Hauert, C., Marotzke, J., Röhl, T., \& Milinski, M. (2013). Intra- and intergenerational discounting in the climate game. Nature Climate Change 3:1025-1028.

Kahneman, D. \& Tversky, A. (1979). Prospect Theory: An Analysis of Decision under Risk. Econometrica 47(2), pp. 263-291. doi: 10.2307/1914185

Kahneman, D. (2003). A Perspective on Judgment and Choice: Mapping Bounded Rationality. American Psychologist 58 (9), pp. 697-720. doi:10.1037/0003-066X.58.9.697

Kahneman, D. (2011). Thinking, Fast and Slow. New York: Farrar, Straus and Giroux Kretzschmar, A. \& Süß, H.M. (2015). A Study on the Training of Complex Problem Solving Competence. Journal of Dynamic Decision Making 1 (4), pp. 1-15. doi:10.11588/jddm.2015.1.15455

Laran, J. \& Salerno, A. (2013). Life-History Strategy, Food Choice, and Caloric Consumption. Psychological Science 24, pp.167-173. doi: 10.1177/0956797612450033

Marshall, G. (2014) Don't Even Think About It: Why Our Brains Are Wired to Ignore Climate Change. Bloomsbury USA.

McCright, A. M., \& Dunlap, R. E. (2011). The politicization of climate change: Political polarization in the American public's views of global warming. The Sociological Quarterly, 52, 155-194.

McNeil, B.J., Pauker, S.G., Sox, H.C. Jr. \& Tversky, A. (1982). On the Elicitation of Preferences for Alternative Therapies. New England Journal of Medicine 306(21), pp. 1259-1262.

doi: 10.1056/NEJM198205273062103 
Micheli, E. G. (2016). Complex Problem Solving and the Theory of Complexity in High School Teaching. Unpublished Dissertation. University of Bergamo, Italy.

Miller, C.H., Lane, L.T., Deatrick, L.M., Young, A.M. \& Potts, K.A. (2007). Psychological Reactance and Promotional Health Messages: The Effects of Controlling Language, Lexical Concreteness, and the Restoration of Freedom. Human Communication Research 33 (2), pp. 219-240. doi: 10.1111/j.1468-2958.2007.00297.x

Nettle, D. (2010). Dying Young and Living Fast: Variation in Life History across English Neighborhoods. Behavioral Ecology 21 (2), pp. 387-395. doi: 10.1093/beheco/arp202 OECD (2014). PISA 2012 results: Creative problem solving: Students' skills in tackling reallife problems (Volume V). PISA, OECD Publishing

Osman, M. (2004). An Evaluation of Dual-process Theories of Reasoning. Psychonomic Bulletin \& Review 11(6), pp. 988-1010. doi:10.3758/BF03196730

Osman, M. (2010). Controlling Uncertainty: Decision Making and Learning in Complex Worlds. Chichester: Wiley-Blackwell.

Osman, M. (2014). Future-Minded: The Psychology of Agency and Control. Basingstoke: Palgrave MacMillen.

Osman, M. (2015). Does our unconscious rule? Psychologist 28 (2), 114-117.

Ravetz, J.R. (2006). Post-normal Science and the Complexity of Transitions towards Sustainability. Ecological Complexity 3 (4), pp. 275-284. doi: 10.1016/j.ecocom.2007.02.001

Sloman, S.A. (1996). The Empirical Case for Two Systems of Reasoning. Psychological Bulletin 119 (1), pp. 3-22. doi: 10.1037/0033-2909.119.1.3

Solso, R. L. (2001). Cognitive Psychology. Boston: Allyn and Bacon 
Sonnleitner, P, König, A. \& Sikharulidze, T. (under review). Computer-based Complex Problems as a Tool to Illustrate Systems Thinking and Confront Learners with Emotions and Uncertainty When Facing Complexity. Environmental Education Research, pp. 1-44

Sonnleitner, P., Keller, U., Martin, R. \& Brunner, M. (2013). Students’ Complex Problemsolving Abilities: Their Structure and Relations to Reasoning Ability and Educational Success. Intelligence 41 (5), pp. 289-305. doi:10.1016/j.intell.2013.05.002

Stanovich, K. E. (2004). The Robot's Rebellion: Finding Meaning the Age of Darwin. Chicago: University of Chicago Press

Stanovich, K. E. (2009). What Intelligence Tests Miss: The Psychology of Rational Thought. Yale: University Press.

Stanovich, K.E. \& West, R.F. (2000). Individual Differences in Reasoning: Implications for the Rationality Debate? Behavioral and Brain Sciences 23, pp. 645-726

Thaler, R.H. \& Sunstein, C.R. (2008). Nudge: Improving Decisions about Health, Wealth, and Happiness. New Haven, London: Yale University Press

Thaler, R.H. (1980). Toward a Positive Theory of Consumer Choice. Journal of Economic Behavior and Organization 1(1), pp. 39-60. doi: 10.1016/0167-2681(80)90051-7

Vester, F. (2012). The Art of Interconnected Thinking: Ideas and Tools for tackling with Complexity. Munich: MCB-Verlag

Wood, B. (2005). Human Evolution: A Very Short Introduction. New York: Oxford University Press 